\title{
COMPARTILHAMENTO DE ARQUIVOS: MELHORIAS PARA COLABORAÇÃO DA INFORMAÇÃO ${ }^{1}$
}

\author{
FILE SHARING: IMPROVEMENT FOR COLLABORATION OF \\ INFORMATION
}

\author{
Grazieli Monteiro Furukawa \\ Universidade Federal de Minas Gerais (UFMG) \\ grafurukas@yahoo.com.br \\ Eduardo Marques Arantes \\ Universidade Federal de Minas Gerais (UFMG) \\ arantes@ufmg.br
}

\begin{abstract}
Resumo
O artigo apresenta as dificuldades apontadas em alguns estudos no que se refere a mudança de comportamento dos profissionais que atuam em equipes multidisciplinares necessária para a adoção da metodologia BIM e de plataformas colaborativas. São identificadas possibilidades de melhorias para a colaboração da informação coletadas a partir dos resultados da pesquisa de mestrado quando foram analisados os registros de utilização da plataforma colaborativa Sisac utilizada para três projetos colaborativos. Os resultados obtidos revelaram dificuldades de interatividades entre a maior parte das disciplinas de projetos. Destaca-se o melhor desempenho das disciplinas de arquitetura, estruturas e sistemas prediais. Constatou-se também a dificuldade de utilização das ferramentas colaborativas em tempo real, sendo estes os principais pontos a serem melhorados pelos profissionais para otimização do processo de projetos e aproveitamento de toda a potencialidade das ferramentas colaborativas e possivelmente o BIM. O estudo apresenta como contribuição o ponto de vista dos usuários coordenadores de projeto e relata a importância de ações eficazes de gerenciamento identificadas também através de análise documental.
\end{abstract}

Palavras-chave: Projeto colaborativo. Colaboração da informação. Ferramenta colaborativa.

\begin{abstract}
The article presents the difficulties pointed out in some studies with regard to changing the behavior of professionals working in multidisciplinary teams required for the adoption of BIM methodology and collaborative platforms. Improvements to the collaboration of information possibilities are identified collected from the master of search results when the records were analyzed using the Sisac collaborative platform for three collaborative projects. The results revealed difficulties interactivity between the most designs disciplines. Noteworthy is the best performance of the disciplines of architecture, structures and building systems. It was also found the difficulty of use of collaborative tools in real time, which are the main points to be improved by professionals to optimize the design process and use the full power of collaborative tools and possibly the BIM. The study presents a contribution point of view of design engineers and users have reported the importance of effective management actions identified also through document analysis.
\end{abstract}

Keywords: Collaborative project. Collaboration information. Collaborative tool.

\footnotetext{
${ }^{1}$ FURUKAWA, G.; ARANTES, E. M. Compartilhamento de arquivos: melhorias para colaboração da informação. In: ENCONTRO BRASILEIRO DE TECNOLOGIA DE INFORMAÇÃO E COMUNICAÇÃO NA CONSTRUÇÃO, 7. 2015, Recife. Anais... Porto Alegre: ANTAC, 2015.
} 


\section{INTRODUÇÃO}

Nos últimos anos a indústria da arquitetura, engenharia e construção civil (AEC) foi marcada pelo surgimento da tecnologia BIM (Building Information Modelling). De acordo com Eastman et al. (2008, p.13), BIM é "uma tecnologia de modelagem e um grupo associado de processos para produção, comunicação e análise do modelo de construção". Meiriño (2013) define o BIM como uma metodologia de trabalho vinculada a sistemas informatizados, que tem por finalidade promover o gerenciamento de todas as etapas de um empreendimento relacionado à construção civil.

No contexto atual o uso do BIM nos escritórios das diferentes especialidades de projetos já é uma realidade. Entretanto, pesquisas recentes apontam algumas dificuldades para implantação da metodologia de trabalho, no que se refere a colaboração da informação. Em 2009 Andrade alertou sobre os principais problemas no desenvolvimento de sistemas BIM que, segundo o autor está no entendimento desses sistemas pelos profissionais da indústria da AEC. O autor argumenta que o BIM enquanto processo de trabalho envolve, sobretudo, a comunicação e a colaboração entre diferentes profissionais e empresas ligadas à AEC. Todavia, o que se observa é que poucas empresas e profissionais que vêm utilizando as ferramentas BIM buscam a padronização e a colaboração.

Para Lino (2012) existe um conjunto de barreiras e limitações que estão a inibir a adoção rápida do BIM na prática profissional. Muitas condicionantes são de natureza técnica e têm sido gradualmente abordadas pelos produtores de software, pelos investigadores e pelas organizações. No entanto, são as questões centradas nas pessoas e nas organizações as que colocam os maiores desafios à implementação do BIM uma vez que a mudança de procedimentos e fluxos de comunicação, responsabilização e confiança, não é facilmente alterável. Entre outros aspetos destaca-se o envolvimento da equipe: ainda é escassa a proliferação desta metodologia entre equipes que conseguem o envolvimento de todos os projetistas. Assim, ao se reduzir logo de partida, o âmbito possível de uma das maiores potencialidades do BIM que é a interação colaborativa, a qual permite lidar com alterações e com incompatibilidades entre especialidades de modo imediato, está-se, naturalmente, a contribuir para a redução da sua relevância e do retorno da tecnologia.

Checcucci (2011) ressalta a importância da etapa de colaboração na aplicação da metodologia BIM. Na Figura 1 a autora apresentou o BIM nas diversas fases do ciclo de vida da edificação através.

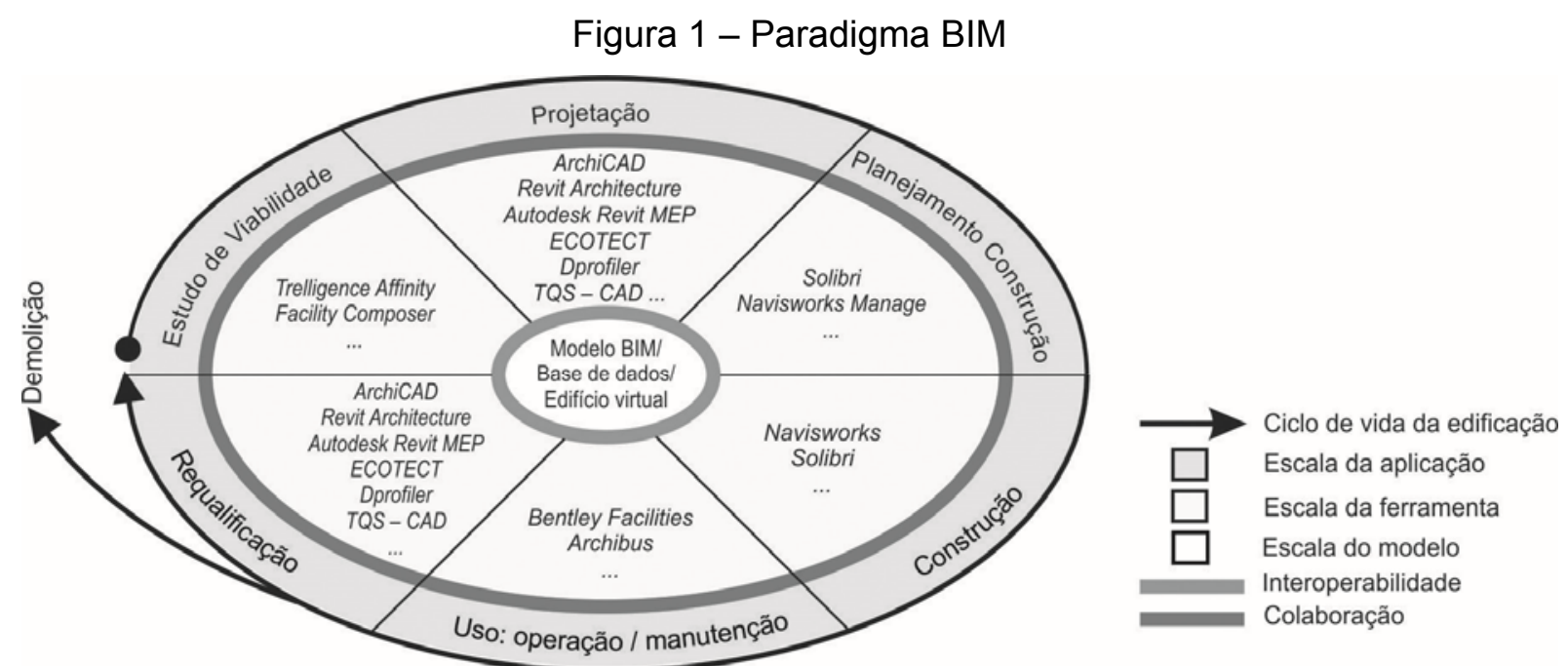

Fonte: Checcucci, 2011 
Para autora a etapa da colaboração se refere à adoção de metodologias de trabalho em equipe multidisciplinar através da análise organizacional do processo de projetos e construção, visto como capacidade de comunicar dados do produto (textos, imagens, planilhas, etc.) através de profissionais de diferentes áreas. O BIM impõe o desenvolvimento de novas formas de colaborar, produzir e compartilhar o conhecimento. Neste contexto, é fundamental a compreensão de que a tecnologia por si só não tem o poder de resolver todos os problemas da gestão da informação durante o ciclo de vida da edificação. Assim, faz-se necessário analisar e atualizar os processos envolvidos, revendo a função de cada ator no processo de modelagem. A edificação deverá ser concebida através da participação multidisciplinar integrada, onde todos tenham a compreensão global do modelo, viabilizando a transferência contínua de conhecimento entre os diversos participantes (CHECCUCCI, 2011).

Ruschel et. al. (2013) realizou um estudo sobre as funcionalidades de algumas ferramentas de colaboração dos modelos BIM. Selecionou-se para estudo, as ferramentas BIM Server e EDMmodelServer que dão suporte à colaboração externa compartilhando modelos BIM no formato aberto (IFC - Industry Foundation Classes). Estas são ferramentas similares, entretanto a primeira é desenvolvida e distribuída como opensourse e a segunda é proprietária. Incluiu-se também uma terceira ferramenta, o conjunto REVIT Serve e Autodesk Project Bluestreak, pois permitem a colaboração interna entre agentes de uma mesma disciplina viabilizando a edição segmentada e distribuída de um mesmo modelo de informação.

Estudos de caso voltados para a colaboração da informação utilizando as ferramentas colaborativas dos modelos BIM ainda são escassos. A aplicação de tais ferramentas está em ascensão no Brasil. Entretanto, já se tem referências consolidadas quanto ao compartilhamento e colaboração de informações para o desenvolvimento de projetos utilizando sistemas colaborativos. As chamadas extranets de projetos já são utilizadas desde a década de 1990. Segundo Coelho (2008) os sistemas colaborativos voltados para a construção civil surgiram na segunda metade da década de 1990, através de empreendimentos conjuntos (joint ventures) de grandes companhias de construção civil, com o objetivo de promover maior produtividade e eficiência no setor.

Ambientes colaborativos computacionais são facilitadores particularmente importantes na adoção de princípios de engenharia simultânea. Em parte, isso se deve a uma maior necessidade de estruturação do fluxo de informações a serem compartilhadas, sobretudo quando são envolvidos múltiplos agentes detentores de conhecimentos e informações tecnológicos específicos (ANDERY \& VEIGA, 2013).

No sentido de fomentar pesquisas futuras o presente trabalho mostra os resultados da investigação de um processo de projeto colaborativo desenvolvido através do Sisac (Sistema de Ambiente Colaborativo). Os dados dessa investigação foram coletados para desenvolvimento da pesquisa de mestrado sobre a aplicação do sistema colaborativo para o desenvolvimento de projetos simultâneos. $O$ volume de dados obtidos somado à possibilidade de estuda-los cruzando de diversas formas possíveis despertou o interesse de utilizar tais informações para responder a questões também levantadas por pesquisadores quanto à utilização do BIM.

Focada neste objetivo, a investigação concentrou-se na análise dos registros de utilização do ambiente colaborativo classificados em ordem cronológica, por usuário, especialidade de projeto e objetivo do acesso (upload ou download). Acredita-se que os resultados dessa pesquisa possam esclarecer questões que vão além da utilização da ferramenta, como uma tendência de comportamento ou organizacional.

O Quadro 1 apresenta uma comparação entre as funcionalidades das ferramentas compartilhadoras de modelos BIM analisadas por Ruschel et. al. (2013) e o Sisac. 
Quadro 1 - Ferramenta compartilhadora

\begin{tabular}{|c|c|c|c|c|}
\hline FUNCIONALIDADES & $\begin{array}{l}\text { REVIT-SERVER } \\
\text { / BLUESTREAK }\end{array}$ & BIM-SERVER & EDMmodelServer & SISAC \\
\hline $\begin{array}{l}\text { Gestão de usuários (Estrutura } \\
\text { hierárquica de administração e } \\
\text { registro de acesso) }\end{array}$ & $x$ & $X$ & $x$ & $x$ \\
\hline $\begin{array}{l}\text { Banco de Dados que consolida } \\
\text { modelos (com controle de } \\
\text { transações) }\end{array}$ & $x$ & $x$ & $X$ & - \\
\hline Controle de versões & $x$ & - & $x$ & $x$ \\
\hline $\begin{array}{l}\text { Compartilhamento de } \\
\text { documentos e relatórios }\end{array}$ & $X^{*}$ & $x$ & $x$ & $x$ \\
\hline Recursos de visualização & $X^{*}$ & $x$ & $x$ & - \\
\hline $\begin{array}{l}\text { Trabalho distribuído em tempo } \\
\text { real }\end{array}$ & $x$ & $x$ & $x$ & $x$ \\
\hline
\end{tabular}

*Nota: Apenas para o Bluestreak e também o único que faz comunicação síncrona entre partes envolvidas

Fonte: Adaptado de Ruschel et. al. (2013)

\section{MÉTODOS E ESTRATÉGIA}

O Sisac é uma extranet de projeto originado com o objetivo de contribuir para pesquisas voltadas para o estudo do fluxo de projetos na engenharia e na arquitetura. Tais estudos se direcionam para o projeto, cobrindo aspectos que vão desde a análise da sua progressão (com ênfase nos seus processos gerenciais e de fluxo de informações) até o estudo de sua natureza, com foco nas características intrínsecas da atividade projetual em arquitetura e engenharia.

Foram investigados três ambientes colaborativos criados para o desenvolvimento de projetos de empreendimentos distintos. Os ambientes colaborativos foram selecionados considerando a similaridade entre os empreendimentos, o volume de registros gerados e o estágio de evolução do processo de projetos. Os estudos dos ambientes denominados como AC1, AC2 e AC3 concentraram-se nos registros de utilização, registros documentais e nos depoimentos cedidos pelos coordenadores de projetos. Conforme mencionado anteriormente os registros de utilização do ambiente colaborativo foram classificados em ordem cronológica, por usuário, especialidade de projeto e objetivo do acesso (upload ou download). A Figura 2 explica a distribuição dos dados da investigação.

O objetivo da investigação é identificar através da análise dos dados e registros coletados os desafios da colaboração da informação, pontuando-os para que sejam tratados pelos gerenciadores de um processo de projeto colaborativo visando a utilização com eficiência de uma plataforma de projeto ou da metodologia BIM. 
Figura 2 - Estratégia da investigação

\section{Ambiente Colaborativo (AC1, AC2 e AC3)}

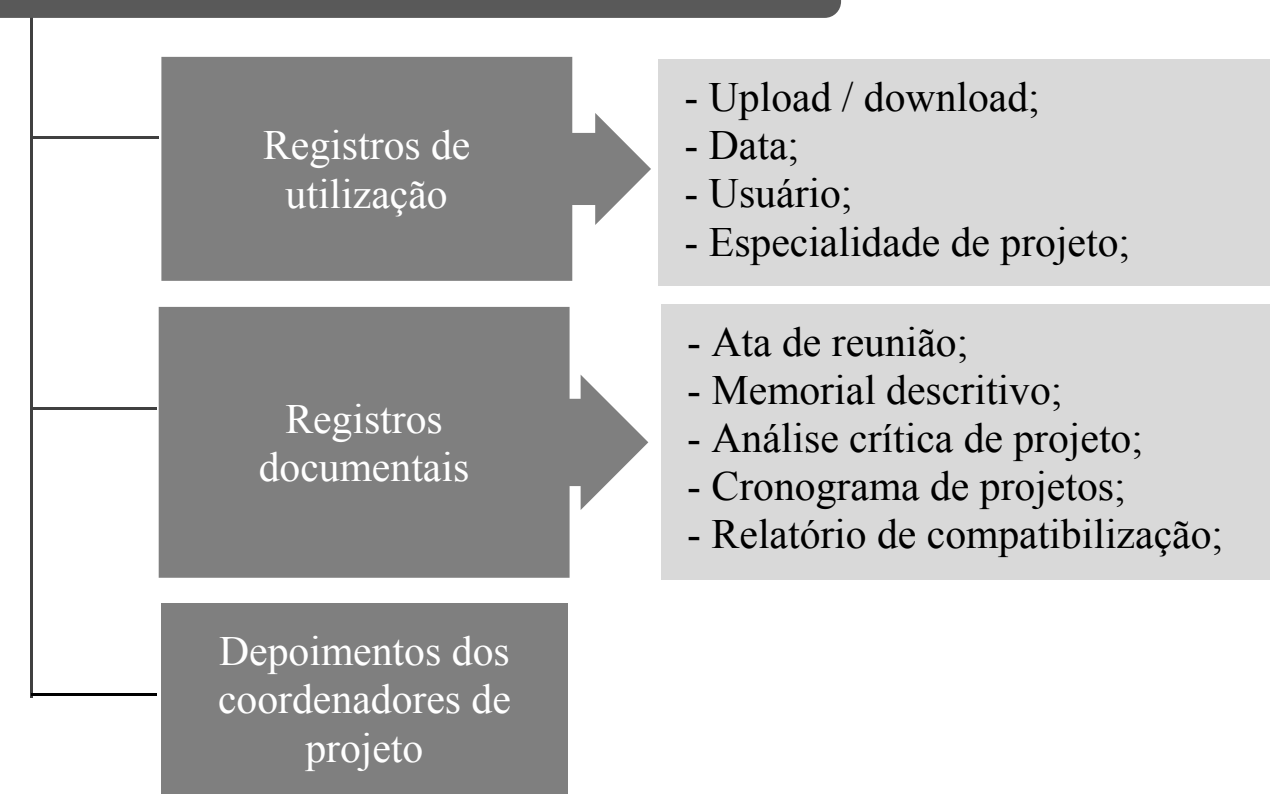

Fonte: Elaboração dos autores (2015)

\section{A COLABORAÇÃO DA INFORMAÇÃO}

Incialmente foi analisado o volume de dados gerados por disciplina de projeto. O Quadro 2 mostra a distribuição percentual dos dados para os três ambientes. Percebe-se que as disciplinas de Arquitetura, Estruturas e Sistemas Prediais tiveram maior representatividade no volume das informações compartilhadas para os três ambientes colaborativos. O Gráfico 1 ilustra esta comparação entre as disciplinas de projeto.

Quadro 2 - Características dos ambientes colaborativos

\begin{tabular}{|c|c|c|c|c|}
\hline \multicolumn{2}{|r|}{$\begin{array}{c}\text { Características do ambiente } \\
\text { colaborativo }\end{array}$} & AC1 & AC2 & AC3 \\
\hline \multicolumn{2}{|c|}{ Total de registros (upload e download) } & 79053 & 5168 & 6280 \\
\hline \multirow{9}{*}{ 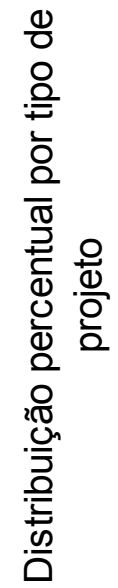 } & Arquitetura & $24,37 \%$ & $26,90 \%$ & $37,96 \%$ \\
\hline & Coordenação & $0,02 \%$ & $3,56 \%$ & $5,51 \%$ \\
\hline & Estruturas & $41,72 \%$ & $10,74 \%$ & $19,18 \%$ \\
\hline & Fundação & $3,53 \%$ & $0,10 \%$ & $9,22 \%$ \\
\hline & Incorporação & $0,48 \%$ & $3,39 \%$ & $0,00 \%$ \\
\hline & Obra & $0,09 \%$ & $0,00 \%$ & $12,15 \%$ \\
\hline & Sistemas Prediais & $22,04 \%$ & $47,00 \%$ & $14,82 \%$ \\
\hline & Vedação & $7,86 \%$ & $6,27 \%$ & $0,00 \%$ \\
\hline & Diversos & $0,00 \%$ & $2,05 \%$ & $1,16 \%$ \\
\hline
\end{tabular}

Fonte: Elaboração dos autores (2013) 
Gráfico 1 - Representação percentual de informações compartilhadas por disciplina

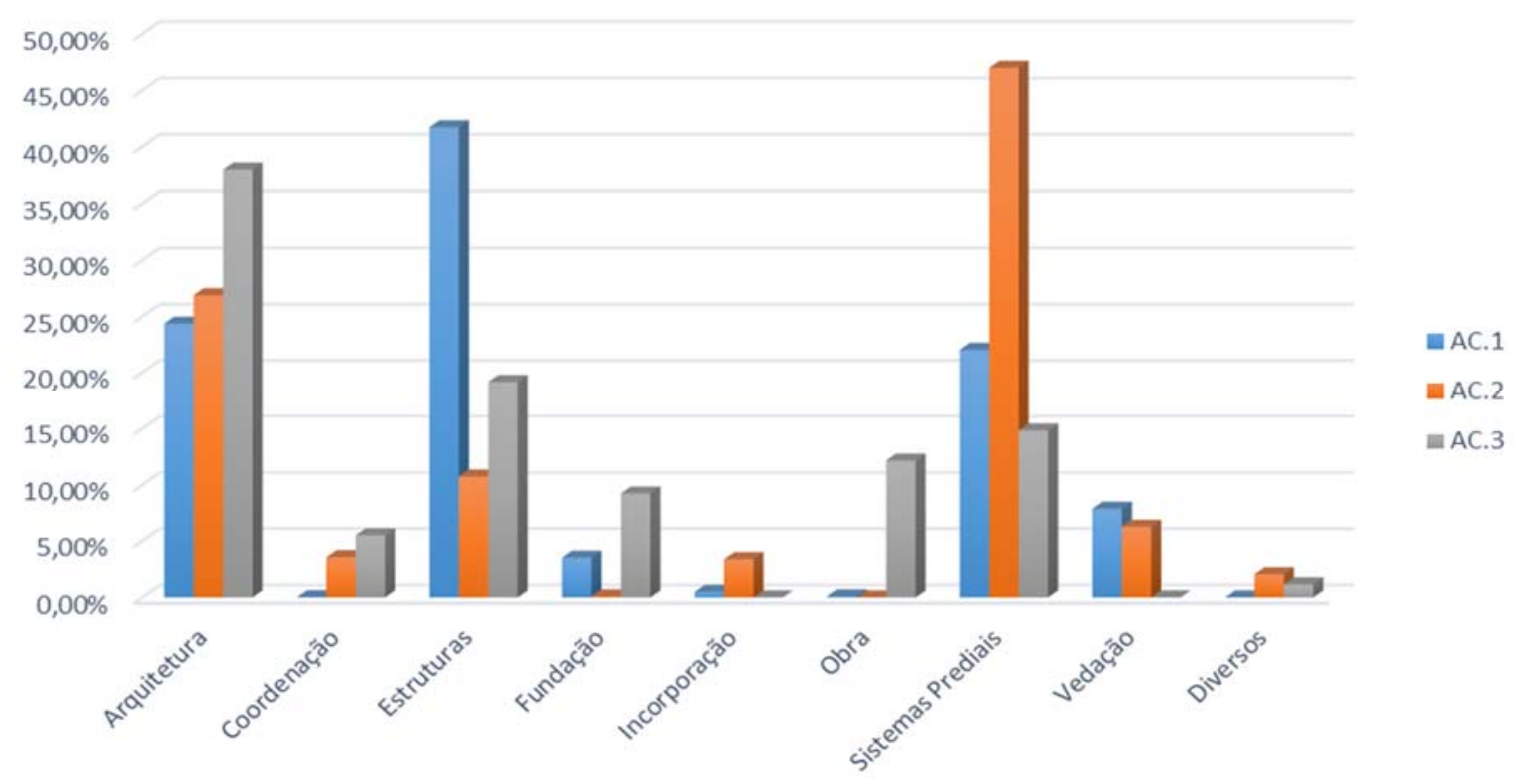

Fonte: Elaboração dos autores (2013)

Os registros das disciplinas de maior representatividade (Arquitetura, Estruturas e Sistemas Prediais) revelaram excesso de dados devido ao elevado número de revisões dos arquivos de projetos. Neste contexto, o excesso de informações tende a dificultar a colaboração. Além disso, as revisões e alterações geradas muitas vezes por incompatibilidade de projetos, ausência de informações, mudança de conceito e demais erros de elaboração demonstram a fragilidade do processo colaborativo. Segundo Checcucci (2011) o BIM impõe que a edificação deverá ser concebida através da participação multidisciplinar integrada, onde todos tenham a compreensão global do modelo, viabilizando a transferência contínua de conhecimento entre os diversos participantes. Além disso, Lino (2012) argumenta que, dadas as características da metodologia BIM, facilmente se conseguem detectar incompatibilidades e conflitos entre os diversos elementos que compõe cada uma das especialidades tornando explícita a interdependência que existe entre as estruturas, a arquitetura, as instalações elétricas ou as instalações mecânicas e hidráulicas, integrando tecnologicamente todos os projetistas. Cita-se, portanto, a redução de retrabalho e dados gerados através da atuação multidisciplinar dos envolvidos como uma melhoria necessária para a colaboração da informação.

O Gráfico 2 ilustra a evolução dos dados dos três ambientes colaborativos ao longo do tempo, identificando-os da seguinte forma: usuários compartilhando informações, registros download/upload e disciplinas de projeto.

Observa-se que os números de usuários e especialidades de projetos não sofreram grandes variações ao longo do tempo e não estavam diretamente relacionados a quantidade de registros gerados. Esta característica pode ser interpretada de forma positiva no que se refere a colaboração da informação, pois revela que ao serem criados novos arquivos os usuários acessaram o ambiente colaborativo para obtê-los, sem a preocupação de aguardar o acúmulo de arquivos postados para realizar o upload. Este comportamento indica ainda a utilização do ambiente colaborativo em tempo real 


\section{TIC2015}

Gráfico 2 - Evolução do AC 1

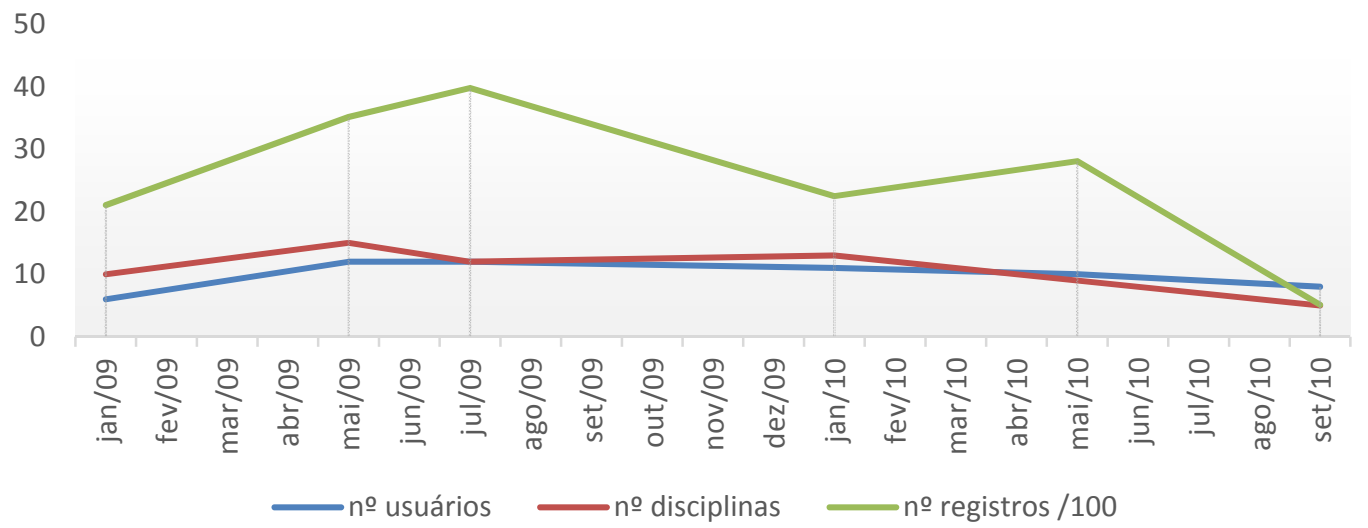

Fonte: Elaboração dos autores (2013)

O acesso à informação em tempo real tende a otimizar o processo de projeto colaborativo por, reduzir o tempo de resposta e agilizar a tomada de decisão. Quando este comportamento está inserido na rotina de trabalho da equipe multidisciplinar, boa parte da potencialidade da ferramenta colaborativa será revertida em benefícios ao processo de projetos. Sendo assim, diante do vasto volume de dados gerados pelos ambientes desta investigação, considerou-se oportuno avaliar o intervalo de acesso dos usuários que, nada mais é que o tempo para acessar um arquivo após postado. Os resultados mais representativos desta avaliação estão apresentados no Quadro 3.

\begin{tabular}{|c|c|c|c|}
\hline \multicolumn{4}{|c|}{ Intervalo de acesso } \\
\hline \multicolumn{3}{|c|}{ Por disciplina } & Por AC \\
\hline \multirow{5}{*}{ AC1 } & Estruturas & 3,33 & \multirow{5}{*}{6,37} \\
\hline & Arquitetura & 6,33 & \\
\hline & Instalações & 8,17 & \\
\hline & Paginação & 11,29 & \\
\hline & Coordenação & 2,75 & \\
\hline \multirow{3}{*}{$\mathrm{AC2}$} & Detalhamento & 16,67 & \multirow{3}{*}{7,70} \\
\hline & Instalações & 4,22 & \\
\hline & Coordenação & 2,2 & \\
\hline \multirow{3}{*}{ AC3 } & Projetista & 10,11 & \multirow{3}{*}{4,59} \\
\hline & Coordenação & 1,34 & \\
\hline & Construtora & 2,33 & \\
\hline
\end{tabular}

Fonte: Elaboração dos autores (2015)

Ao avaliar o intervalo de acesso entre os usuários foram consideradas também as relações interativas entre eles. $\mathrm{Na}$ ocasião observou-se que o $\mathrm{AC} 1$ revelou ter maior interatividade 
entre cinco diferentes disciplinas de projetos, se comparado aos demais, que apresentaram interatividade apenas entre 3 disciplinas. A pouca interatividade entre as disciplinas pode ser considerada uma segunda melhoria para a colaboração da informação. Coelho (2008) ressalta que a adoção de sistemas BIM refere-se à adoção de novos fluxos de trabalho envolvendo ambiente colaborativo e planejamento nas fases iniciais do projeto. $O$ novo modelo de colaboração envolve recursos avançados de visualização, aliados à transferência contínua de conhecimento entre os diversos agentes participantes do processo de projeto (projetistas, construtores, contratantes, consultores, etc.).

Para o AC2 devem ser considerados importantes fatos extraídos da análise documental. Os arquivos da disciplina de arquitetura foram postados numa única data e após esta não foram geradas novas revisões. Não foi possível, portanto visualizar a interação dessa disciplina com as demais. Destaca-se também como melhoria a necessidade de utilização do ambiente colaborativo para desenvolvimento todas as especialidades de projetos. Para Lino (2012) o conceito BIM assenta, essencialmente, numa metodologia de partilha da informação entre todos os intervenientes.

O AC3 apresentou o diferencial na estrutura do ambiente colaborativo, quando o administrador do ambiente optou por distribuir os arquivos em apenas três pastas distintas de projetos (aqui denominadas como disciplinas) sendo elas: Projetistas, Coordenação e Construtora. Obviamente que tal distribuição restringiu a visualização da interatividade entre as disciplinas. Ainda assim, vale destacar que o AC3 apresentou o menor intervalo de acesso da investigação.

Ressalta-se o comportamento em comum observado nos três ambientes colaborativos: a participação relevante dos responsáveis pela coordenação dos projetos, que apresentaram o menor intervalo de acesso das equipes multidisciplinares.

De forma geral, os números do Quadro 3 mostram que os usuários demoram no mínimo 1,34 dias para fazer um download de um arquivo, o que em termos de colaboração, não pode ser considerado acesso em tempo real. Para isso o intervalo de acesso deveria ser próximo de zero, sendo esta uma melhoria necessária para a efetiva colaboração da informação no processo de projetos. Sabe-se que a plataforma colaborativa analisada oferece essa possibilidade ao usuário. Nos ambientes analisados foram identificados usuários que utilizaram as vantagens da colaboração para obter arquivos em tempo real, porém, não foram em número suficiente para impactar no resultado geral. A opção de utilizar a plataforma colaborativa como se deve, aproveitando suas potencialidades, fica condicionada sobretudo a mudança na cultura de trabalho do usuário. Neste sentido, a análise documental a seguir identifica ações de gerenciamento que promovam a colaboração da informação no processo de projeto.

\subsection{Análise documental}

Durante o processo de desenvolvimento dos projetos foram gerados documentos que revelaram a comunicação entre os envolvidos. Alguns foram selecionados para análise em razão de sua importância contribuição para a colaboração da informação.

Nos registros documentais do AC1 foram identificadas algumas ações gerenciais que promovem a colaboração da informação. Atas de reunião e cronogramas de projetos que apresentam informações das versões atualizadas dos projetos, observações para compatibilização e especificações de materiais são disponibilizados na plataforma colaborativa e tal ação é documentada e divulgada a todos os participantes nas reuniões presenciais de projeto. A Figura 3 mostra um trecho extraído de uma ata de reunião compartilhada no ambiente colaborativo. 
Figura 3 - Trecho da Ata de Reunião: citações do SISAC no processo de projetos - AC1

\begin{tabular}{|c|c|c|c|c|c|}
\hline \multicolumn{2}{|c|}{ Item } & Assunto & Responsável & Data & Status \\
\hline \multirow[t]{6}{*}{01} & \multicolumn{5}{|c|}{ Coordenação } \\
\hline & \multicolumn{5}{|c|}{01.01 Coordenação de Projetos e interface com } \\
\hline & 01.01 .04 & $\begin{array}{l}\text { solicitou uma revisão da } \\
\text { organização das pastas em Sisac para facilitar } \\
\text { consulta. e irão avaliar possivel } \\
\text { alteração das pastas após a finalização da } \\
\text { entrega de projetos da fase 01; }\end{array}$ & & $\begin{array}{l}\text { Após } \\
30 / 05 / 09\end{array}$ & \\
\hline & \multicolumn{5}{|c|}{ 01.02 Rotina de trabalho e Planejamento para finalização dos projetos } \\
\hline & 01.02 .01 & $\begin{array}{l}\text { deverá elaborar o cronograma de } \\
\text { entregas da fase } 01 \text { conforme definições em } \\
\text { reunião e informar data e pauta da próxima } \\
\text { reunião - deverá cadastrar em Sisac; }\end{array}$ & & $27 / 04 / 09$ & \\
\hline & 01.02 .02 & $\begin{array}{l}\text { deverá elaborar cronograma da fase } 02 \\
\text { com etapas e reuniões definidas, e enviar para } \\
\text { aprovação dos projetistas e em seguida } \\
\text { cadastrar em Sisac: }\end{array}$ & & $15 / 05 / 09$ & \\
\hline \multirow[t]{3}{*}{02} & \multicolumn{5}{|c|}{ Situação dos projetos e Planejamento para finalização da fase 01} \\
\hline & \multicolumn{5}{|c|}{02.01 Pavimentos tipo torres $1,2,3,8$} \\
\hline & 02.01 .01 & $\begin{array}{l}\text { deverá cadastrar em Sisac até } 23 / 04 / 09 \\
\text { as matrizes de fôrmas do pavimento tipo das } \\
\text { torres } 1,2,3,8 \text {; }\end{array}$ & & $23 / 04 / 09$ & \\
\hline
\end{tabular}

Fonte: Ata da reunião do AC1. Adaptação dos autores (2013).

No processo de projetos do AC2 destaca-se a contribuição dos cronogramas de projetos e análises críticas de projetos conforme mostrado na Figura 4.

Figura 4 - Análise crítica do projeto elétrico: solicitação de compatibilização com outras especialidades de projeto

\section{PLANILHA PARA ANALISE CRÍTICA DE PROJETOS \\ Data: $01 / 12 / 08$}

DEFICIÊNCIA EM TERMOS DE INFORMAÇÕES PARA PERMITIR A ANALISE:

\section{MODIFICAÇÕES E ADAPTAÇÕES NECESSÁRIAS:}

TORRE 3:

1. Colocar dimensões das caixas $(2 \times 4,4 \times 4)$.

2. Colocar ponto para evaporadora (ver executivo).

3. Marcar iluminação de emergência e sensor de presença nas circulações conforme última revisão do executivo no SISAC. Transferir os pontos da prancha 34 para a prancha 29 (para não corrermos o risco da obra não executar)

4. Transferir posição do conjunto de tomadas $110 \mathrm{~V}$ e $220 \mathrm{~V}$ conforme projeto executivo.

5. Colocar duas cigarras.

6. A posição da tomada de exaustão no banho de empregada está errada. Veja executivo.

OBSERVAÇÕES GERAIS:

ANALISADO PRANCHA 34-EL-65 - Revisão 1

Fonte: Análise crítica de projeto do AC 2. Adaptação dos autores (2013). 
Tais documentos vinculavam a validação de projetos, prazos para entrega e informações da concepção do empreendimento ao que era divulgado na plataforma colaborativa. Acompanhando as atualizações realizadas aos documentos, os memoriais descritivos passaram por revisões frequentes para se obter a definição real do produto e todas as suas versões foram divulgadas na plataforma colaborativa conforme eram geradas.

Os documentos do AC3 apresentaram informações relevantes referentes à concepção do empreendimento. Ainda que a análise do ambiente colaborativo tenha mostrado o desenvolvimento dos projetos arquitetônicos sem a utilização da plataforma colaborativa, as atas de reunião apresentaram discussões detalhadas para especificação de materiais de acabamento e conforto ambiental da edificação, como aquecimento de água e fornecimento de energia. Nesse processo de projetos destaca-se ainda a qualidade das informações repassadas em relatórios de compatibilização, que tendem a minimizar erros de projeto e contribuir para a qualidade do produto final.

Os documentos analisados apresentaram importantes ações dos coordenadores no sentido de promover a utilização da plataforma colaborativa para o desenvolvimento dos projetos. A atuação do coordenador de projetos neste processo é imprescindível para o sucesso da adoção de uma ferramenta colaborativa, esta deve estar consolidada na cultura das equipes e das organizações para a total eficácia do processo de projetos.

\subsection{Depoimentos dos coordenadores de projetos}

Considerada a relevante atuação do coordenador de projetos mencionada anteriormente, foram incluídos na investigação os depoimentos de tais profissionais dos ambientes estudados quanto a utilização das ferrametas colaborativas.

De formar geral os depoimentos foram unânimes em apoiar a utilização de ferramentas colaborativas no processo. Numa abordagem mais ampla, os coordenados entendem ser fundamental o papel de tais ferramentas para a evolução da indústria da AEC.

O Quadro 4 apresenta as principais vantagens da colaboração da informação apontadas por cada coordenador de projeto.

Quadro 4 - Vantagens da colaboração segundo os coordenadores de projeto.

\begin{tabular}{lccc} 
Vantagens da colaboração & AC1 & AC2 & AC3 \\
Acesso à informação & - & X & X \\
\hline $\begin{array}{l}\text { Tomada de decisão } \\
\text { Agilidade nas reuniões de } \\
\text { projeto }\end{array}$ & X & X & X \\
\hline $\begin{array}{l}\text { Histórico de informações } \\
\text { preservado }\end{array}$ & $\mathrm{X}$ & $\mathrm{X}$ & $\mathrm{X}$ \\
\hline $\begin{array}{l}\text { Organização do processo de } \\
\text { projeto }\end{array}$ & - & - & $\mathrm{X}$ \\
\hline $\begin{array}{l}\text { Confiabilidade das } \\
\text { informações }\end{array}$ & $\mathrm{X}$ & $\mathrm{X}$ & $\mathrm{X}$
\end{tabular}

Fonte: Elaboração dos autores (2015). 
As dificuldades apontadas pelos coordenadores de projetos também foram semelhantes. Todos ressaltaram como principal desafio para implantação da ferramenta colaborativa a mudança na metodologia de trabalho das equipes. A fragmentação do processo também foi mencionada como um obstáculo, que muitas vezes abrange dificuldades de localização.

Para vencer as dificuldades, os coordenadores também foram unânimes ao mencionar a necessidade de abordagens gerenciais na promoção da colaboração da informação.

\section{CONCLUSÕES}

Os dados da investigação identificaram três melhorias necessárias para a colaboração da informação:

- Redução de retrabalho e dados gerados através da atuação multidisciplinar dos envolvidos;

- Promover a interatividade entre as diferentes disciplinas de projetos;

- Utilização do ambiente colaborativo para desenvolvimento todas as especialidades de projetos;

- Redução do intervalo de acesso dos arquivos;

A investigação revelou através da análise do volume de dados por disciplina e do intervalo de acesso que os usuários das disciplinas de arquitetura, estruturas e sistemas prediais exercem respectivamente atividades colaborativas de maior representatividade e interatividade no processo de projetos.

Esta informação não está relacionada ao fato de serem disciplinas que geram maior quantidade de registros, pois foi observado que a quantidade de usuários não foi impactada pela variação na quantidade de registros gerados. Sendo considerada, portanto, uma característica típica e bom hábito dos profissionais dessas disciplinas fazer uso regular da plataforma colaborativa para compartilhamento de informações.

A análise do tempo gasto pelos usuários para visualizar um arquivo disponibilizado (intervalo de acesso) no ambiente mostrou que eles não foram acessados em tempo real. Ainda que o menor intervalo de acesso registrado tenha sido consideravelmente baixo (1,34), a ferramenta colaborativa não foi utilizada em sua máxima potencialidade. $\mathrm{Na}$ ocasião observou-se ainda a baixa interatividade entre a vasta variedade de disciplinas envolvidas no processo, exceto as de arquitetura, estruturas e sistemas prediais como já destacadas anteriormente.

Os registros documentais e depoimentos dos coordenadores identificam pontos positivos na utilização do ambiente e reforçaram a importância de ações gerenciais concomitantes com a utilização da ferramenta colaborativa no sentido de obter todas as vantagens e benefícios de um processo colaborativo.

Diante do exposto é possível afirmar que, ainda que a indústria da AEC esteja evoluindo com uso de tecnologias avançadas que promovam a melhorias dos processos de projeto, os profissionais do setor ainda precisam melhorar 0 desempenho de suas atividades colaborativas. As ferramentas precisam ser utilizadas em todas as suas funcionalidades explorando ao máximo suas potencialidades. As estruturas organizações ainda encontram dificuldades na fragmentação do processo de projetos.

Constatou-se que a ação de divulgar a informação já está consolidada entre as 
equipes, haja vista a considerável quantidade de arquivos nos ambientes e a variedade de seus conteúdos e formatos. A utilização de ambientes colaborativos para compartilhamento de informações já faz parte da cultura das organizações. Entretanto, a busca pela informação em tempo real precisa evoluir. Em processos amplos como os analisados, a variedade de informações geradas num curto espaço de tempo é grande. Para acompanhar este fluxo de informações, as ferramentas colaborativas oferecem os recursos necessários, cabem aos profissionais utilizá-los.

Para estudos futuros, sugere-se a análise das interfaces existentes entre a tecnologia BIM e as extranets no que se refere à migração de documentos das especialidades de projeto entre os ambientes colaborativos.

\section{REFERÊNCIAS}

ANDERY, P. R.; VEIGA, A. C. R. Considerações sobre o gerenciamento de projetos complexos: o caso de exposições museográficas. CONSTRUINDO, v. 5, n. 02, 2013.

ANDRADE, M. L. V. X.; RUSCHEL, R. C. Interoperabilidade entre ArchiCAD e REVIT por meio do formato IFC. 2009. IV ENCONTRO NACIONAL DE TECNOLOGIA DA INFORMAÇÃO E COMUNICAÇÃO NA CONSTRUÇÃO CIVIL. Rio de Janeiro, RJ, Brasil, 17 a 19 de Junho de 2009.

CHECCUCCI, E. S; PEREIRA, A. P. C.; AMORIM, A. L. Colaboração e Interoperabilidade no contexto da Modelagem da Informação da Construção (BIM) 2011.

COELHO, S. S. Coordenação de projetos de edifícios com emprego de sistemas colaborativos baseados em software livre. 2008. 148 f. Dissertação (Mestrado em Engenharia Civil) - Faculdade de Engenharia da Universidade Federal de São Carlos, São Carlos, SP, 2008.

COELHO, S. S. Modelagem de Informações para Construção (BIM) e ambientes colaborativos para gestão de projetos na construção civil (2008). Disponível

em:<http://www2.pelotas.ifsul.edu.br/gpacc/BIM/referencias/COELHO_2008.pdf>. Acesso em: 27 ago. 2015.

EASTMAM, C. TEICHOLZ, P. SACKS, R. LISTON, K. HANDBOOK, B. I. M. A Guide to Building Information Modeling for Owners, Managers, Designers, Engineers and Contractors. 2008.

LINO, J. C.; AZENHA, M.; LOURENÇO, P. Integração da Metodologia BIM na Engenharia de Estruturas. BE2012-ENCONTRO NACIONAL BETÃO ESTRUTURAL, 2012.

MEIRIÑO, M. J. Aspectos da implantação de ferramentas BIM em empresas de projetos relacionados à construção civil. CONGRESSO NACIONAL DE EXCELÊNCIA EM GESTÃO 2013. 